\title{
Reprogramming protein kinase substrate specificity through synthetic mutations
}

Joshua M. Lubner ${ }^{1 *}$, George M. Church ${ }^{2,3}$, Michael F. Chou ${ }^{2,3}$ \& Daniel Schwartz ${ }^{1 *}$

${ }^{1}$ Department of Physiology and Neurobiology, University of Connecticut, Storrs, CT 06269, USA.

${ }^{2}$ Department of Genetics, Harvard Medical School, Boston, MA 02115, USA.

${ }^{3}$ Wyss Institute for Biologically Inspired Engineering, Boston, MA 02115, USA.

${ }^{*}$ Corresponding author

Joshua M. Lubner

75 North Eagleville Rd.

Unit 3156

Storrs, CT 06269

University of Connecticut

joshua.lubner@uconn.edu
Daniel Schwartz

75 North Eagleville Rd.

Unit 3156

Storrs, CT 06269

University of Connecticut

daniel.schwartz@uconn.edu 
1 Protein kinase specificity is largely imparted through substrate binding pocket motifs.

2 Missense mutations in these regions are frequently associated with human disease, and

3 in some cases can alter substrate specificity. However, current efforts at decoding the

4 influence of mutations on substrate specificity have been focused on disease-associated

5 mutations. Here, we adapted the Proteomic Peptide Library (ProPeL) approach for

6 determining kinase specificity to the task of exploring structure-function relationships in

7 kinase specificity by interrogating the effects of synthetic mutation. We established a

8 specificity model for the wild-type DYRK1A kinase with unprecedented resolution. Using

9 existing crystallographic and sequence homology data, we rationally designed mutations

10 that precisely reprogrammed the DYRK1A kinase at the $P+1$ position to mimic the

11 substrate preferences of a related kinase, CK II. This study illustrates a new synthetic

12 biological approach to reprogram kinase specificity by design, and a powerful new

13 paradigm to investigate structure-function relationships underpinning kinase substrate 14 specificity. 


\section{INTRODUCTION}

16 Through their role in the covalent transfer of phosphate from a donor ATP molecule to a

17 phosphoacceptor serine, threonine or tyrosine in a substrate protein, protein kinases in

18 eukaryotes play key roles in cellular signal transduction, and function as gatekeepers for

19 important events such as cell cycle checkpoints, apoptosis, and the immune response $(1,2)$.

20 There are several levels of specificity that allow an individual protein kinase to navigate the

21 daunting number of potential substrates, target the correct subset of proteins, and the correct

22 residues within the appropriate protein for phosphorylation. Beyond temporal and spatial co-

23 localization, protein kinases also attain substrate specificity through pattern recognition of

24 distinctive residues proximal to the phosphoacceptor residue (the "P-site"). This pattern is

25 referred to as a kinase specificity motif (or simply "motif"), and is a model of substrates that are

26 compatible with the kinase's substrate binding pocket and can thus be phosphorylated. Motifs

27 are primarily inferred from known physiological substrates (3), and are sometimes modeled as a

28 string of allowable residues, as a position weight matrix, or as a combination of these. The

29 presumed motif is a well-established starting point for in silico prediction of putative

30 substrates (4); however, for nearly all protein kinases, the numbers of known substrates are

31 very few in number resulting in poorly defined, low-resolution motif models.

32 Recently, Creixell and colleagues demonstrated several cancer mutations within kinase

33 domains that modulated catalytic activity, and in some cases altered substrate specificity (5).

34 These results, along with previous work that traced evolutionary changes in substrate specificity

35 to amino acid substitutions (6), and the identification of potential specificity-determining

36 positions $(7,8)$, suggest that a thorough investigation of amino acid structure-function

37 relationships will be necessary to achieve a principled understanding of kinase specificity. At

38 present, these studies have largely evaluated individual, naturally occurring kinase mutations. In 
39 this work, we sought to explore the potential for rational reprogramming of kinase substrate

40 specificity through multiple directed synthetic mutations.

41 Here, we used the Proteomic Peptide Library (ProPeL) method (9) to accurately

42 measure the specific motifs of both wild-type and mutated kinases (Fig. 1). Using this approach,

43 first a heterologous kinase of interest is expressed in E. coli. The kinase phosphorylates

44 bacterial proteins consistent with its endogenous kinase specificity motif. The extremely low

45 activity of serine/threonine/tyrosine kinases and phosphatases in E. coli (10) allows for a high

46 signal-to-noise ratio, and the absence of confounding human kinase cascades ensures a direct

47 link between expressed kinase and observed phosphorylation event. After cell lysis and

48 proteolysis, the resulting phosphopeptides are identified by tandem mass spectrometry. This

49 can provide hundreds to thousands of kinase-specific phosphopeptides from which a

50 high-resolution motif model is generated. In this case, the motif model is a position weight matrix

51 with constant residues at one or more positions, which are easily visualized using the

52 pLogo (11) graphical representation. That these bacterial substrates are not physiological is

53 irrelevant - the identified motif can be used to accurately model kinase substrate specificity, and

54 predict human substrates (9). Here, we have repeatedly utilized ProPeL to generate and

55 compare motifs for wildtype and synthetic mutant kinases.

56 We chose the Down's syndrome associated Dual specificity tyrosine-phosphorylation-

57 regulated kinase $1 \mathrm{~A}$ (DYRK1A) to act as a model kinase. Although the number of known human

58 DYRK1A substrates is low (only 31, (12)), the specificity motif for wild-type DYRK1A has been

59 partially characterized as including basophilic determinants, and a preference in the $\mathrm{P}+1$

60 position for proline $(13,14)$, where $P+n$ denotes the $n$th residue towards the $C$-terminus of the

61 phosphoacceptor P-site, and P-n denotes the nth residue towards the $\mathrm{N}$-terminus.

62 Mechanistically, the region of the substrate binding pocket spanning the conserved DFG and 
63 APE residues within the kinase sub-domains VII - VIII is termed the "activation segment", and

64 has been implicated through X-ray crystallography to confer substrate specificity by interacting

65 with the amino acids flanking the substrate's P-site (reviewed in Kannan and Neuwald,

$662004,(15))$. DYRK1A is a member of the CMGC (CDK/MAPK/GSK3/CLLK) kinase family, and it

67 has been suggested that the $P+1$ proline specificity typical of this family is imparted by a

68 hydrogen bond with a CMGC-conserved arginine in the activation segment (Fig. 2A, (15, 16)).

69 Given this model, we hypothesized that disrupting this hydrogen bond would reduce DYRK1A's

70 preference for proline at the substrate's $P+1$ position. An interesting exception to the CMGC

71 family $\mathrm{P}+1$ proline preference is Casein kinase II (CK II), which prefers acidic residues at

72 position $\mathrm{P}+1$ (Fig. S1, $(9,17)$ ). At the CMGC-conserved arginine position (R328 in DYRK1A),

73 CK II instead codes for lysine (residue K198, (15)). Therefore, we predicted that the mutant

74 DYRK1A $\mathrm{A}^{\mathrm{R} 328 \mathrm{~K}}$ (mimicking $\mathrm{CK}$ II at the CMGC arginine position) would re-position the lysine side-

75 chain $\varepsilon$-amino group to allow for an electrostatic interaction with substrates containing a $P+1$

76 acidic residue.

77 In this work, we demonstrate the ability to generate kinase specificity models of

78 unprecedented resolution using the ProPeL method. Using existing structural data and

79 sequence homology, we successfully engineered the DYRK1A kinase to exhibit an unnatural

80 substrate specificity, using both individual and multiple directed mutations. Overall, this study

81 illustrates the effects of synthetic activation segment mutations upon substrate specificity, and

82 introduces a new approach for the rational creation of designer kinases.

83

\section{RESULTS}

85 High-resolution determination of wild-type DYRK1A substrate specificity 
86 Before attempting to reprogram DYRK1A, we first needed to create a sufficiently high-resolution

87 model of wild-type DYRK1A substrate specificity to serve as a reference. We created bacterial

88 expression constructs to express (1) a catalytic domain truncation of human DYRK1A (N137 -

89 S496, referred to herein as DYRK1A ${ }^{\mathrm{WT}}$ ); and (2) a catalytically inactive DYRK1A triple mutant

$90 \mathrm{~K} 188 \mathrm{R} / \mathrm{D} 287 \mathrm{~N} / \mathrm{D} 307 \mathrm{~N}$ to function as a kinase dead negative control (referred to herein as

91 DYRK1 $\left.A^{K D}\right)$. Both kinases express robustly in the C41(DE3) E.coli strain as evaluated by

92 western blotting (Fig. S2, lanes 1-3). Using the in-gel phosphoprotein stain Pro-Q Diamond, we

93 observed DYRK1 $\mathrm{A}^{\mathrm{WT}}$ exhibits strong autophosphorylation, and efficiently phosphorylate E. coli

94 proteins throughout the gel and thus across the proteome. Importantly, DYRK1A ${ }^{\mathrm{KD}}$ shows

95 neither autophosphorylation, nor substrate phoshorylation (Fig. S3A and S3B, lanes 1-3).

96 Using the ProPeL method, we identified 6,059 unique DYRK1A phosphorylation sites

97 (3,089 pSer, 2,412 pThr, and 558 pTyr) on bacterial proteins. Note that this data set is an order

98 of magnitude larger than that of the human kinase with the largest number of known natural

99 substrates (CDK2, with 514 substrates (12)). Therefore, DYRK1A ${ }^{\text {WT }}$ ProPeL data results in

100 high-resolution motifs that offer a dramatic improvement over those created with only the 18

101 known serine and 13 known threonine literature sites (visualized with the pLogo tool in Fig. 2B,

102 2C and Fig. S4A, S4B). In agreement with previous studies $(13,14)$, DYRK1A ${ }^{\text {WT }}$ exhibited a

103 strong preference for proline in the $\mathrm{P}+1$ position, and basic residues (particularly arginine) in the

104 upstream positions, which together form the optimal consensus sequence RxxS*P (Fig. 2C).

105 Our data confirms the recent suggestion in the literature that DYRK1A can phosphorylate

106 substrates with alternative residues in the $\mathrm{P}+1$ position (14); beyond a strong $\mathrm{P}+1$ preference for

107 proline, DYRK1A ${ }^{\text {WT }}$ also efficiently phosphorylates substrates with hydrophobic residues

108 (particularly valine and alanine), or arginine in the $\mathrm{P}+1$ position. While these residues are

109 statistically significant in the $\mathrm{P}+1$ position for serine P-sites, they fail to occur at statistical 
110 significance for threonine P-sites (Fig. S4B). Threonine P-site substrate specificity, therefore,

111 may be more dependent on the $\mathrm{P}+1$ proline than are serine $\mathrm{P}$-site substrates. There is also a

112 strong, previously unreported hydrophobic cluster present at $\mathrm{P}+2$ for both serine and threonine

113 substrates. DYRK1A does not exhibit a significant phosphotyrosine motif (Fig. S4C), however

114 our 558 unique tyrosine phosphorylation sites in E. coli clearly indicate that DYRK1A is capable

115 of phosphorylating tyrosine substrates in trans, and that phosphotyrosine activity is not

116 restricted to autophosphorylation, as previously thought $(13,18)$.

117 Using the pLogo tool and an internal version of the motif- $x$ program (19, 20), we

118 evaluated dependence between motif positions. It is important to note that while we identify

119 tryptic peptides by tandem mass spectrometry, the kinase-substrate interaction that produced

120 the phosphorylation event occurred in the context of full-length substrate proteins. Therefore, we

121 are able to map tryptic fragments back to the known E. coli proteome and extend sequences

122 beyond the detected tryptic fragment, allowing us to analyze the presence (or absence) of

123 multiple upstream basic residues. This analysis revealed that although there is a strong

124 correlation between $\mathrm{P}+1$ proline and upstream basic residues (Fig. S5A, S5B), there is no

125 significant correlation between multiple upstream basic residues (Fig. S5C to S5F). Therefore,

126 the optimal motif sequence is actually $R x x S^{*} P$, and not $R R R R x S^{*} P$, which is the broad motif

127 without respect to any interdependent substrate residues. Substrates conforming to $R \times S^{*} P$,

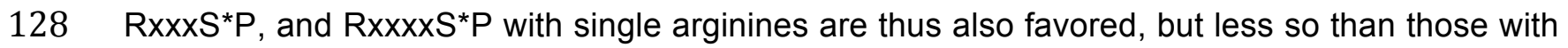

129 RxxS*P. We note that multiple arginines are actually not favored for substrate recognition,

130 although they do not appear to be clearly disfavored either. The complete list of statistically

131 significant motif classes for DYRK1A (and all kinases within this study) identified by motif- $x$ can

132 be found in Table S2. 
133 To verify the specificity of our DYRK1A model amongst other known kinases, we

134 performed an in silico analysis using our high-resolution DYRK1A motif. We scored known

135 human DYRK1A substrates, an equivalent number of substrates randomly selected from the

136 human proteome, as well as known substrates for other kinases from the remaining

137 serine/threonine kinase families (12). Our motif was able to accurately discriminate known

138 DYRK1A substrates from random substrates and also performed well in discriminating against

139 non-DYRK1A kinase substrates (Fig. S6).

141 Mutation of Q323 reduces wild-type DYRK1A P+1 proline preference

142 As introduced earlier, DYRK1A P+1 substrate preference is hypothesized to be imparted by a

143 hydrogen bond between the side-chain nitrogen of the CMGC-conserved arginine (DYRK1A ${ }^{R 328}$ )

144 and the main-chain oxygen of a non-glycine residue (DYRK1 $A^{\text {Q323})}$ undergoing torsional strain

145 (Fig. 2A, $(15,16)$ ). This hydrogen bond should thus neutralize the main-chain oxygen's dipole

146 moment and facilitate interaction with substrates containing a proline at $P+1$. The loss of the

147 bulky glutamine side-chain in a glutamine to glycine mutant (DYRK1 ${ }^{Q 323 G}$ ) could theoretically

148 relieve torsional strain and re-position the kinase's main-chain oxygen. This could destabilize

149 the hydrogen bond, and ultimately result in a reduced $\mathrm{P}+1$ proline preference.

150 Using ProPeL to determine DYRK1A ${ }^{Q 323 G}$ specificity, we identified 1,579 unique

151 phosphorylation sites (829 pSer, 678 pThr, and 72 pTyr). Consistent with our hypothesis, motif

152 visualization with pLogo revealed a significant reduction in $\mathrm{P}+1$ proline preference (Fig. $3 \mathrm{~A}$ and

153 Fig. S7A). For DYRK1A ${ }^{W T}$, hydrophobic residues constitute secondary determinants in the P+1

154 position (particularly for serine substrates). For our engineered DYRK1A ${ }^{\text {Q323G }}$ mutant acting

155 upon serine P-sites, alanine, not proline, becomes the most statistically significant substrate 
156 residue at position $\mathrm{P}+1$. For threonine $\mathrm{P}$-sites, proline is still the most statistically significant

157 residue at position $P+1$, but is greatly reduced when compared to DYRK1A ${ }^{W T}$.

159 Mutation of R328 mimicks CK II P+1 acidic preference

160 As discussed above, we hypothesized that creation of a DYRK1A variant that mimics CK II at

161 the CMGC-conserved arginine position (DYRK1 $A^{R 328 K}$ ) would reprogram the $P+1$ preference

162 from proline to acidic residues. Using ProPeL to analyze DYRK1A ${ }^{R 28 K}$, we identified 756 unique

163 phosphorylation sites (399 pSer, 327 pThr, and 30 pTyr). Agreeing with our hypothesis, the

164 DYRK1A $A^{R 328 K}$ mutant does show a significant increase in acidic $P+1$ preference for both serine

165 and threonine P-site substrates (Fig. 3B and Fig. S7B). We note that phosphorylation of

166 substrates with $P+1$ proline occur at similar levels to DYRK1 $A^{W T}$, suggesting that the

167 substitution of arginine with lysine at the CMGC-conserved arginine position is still capable of

168 forming the putative hydrogen bond. In the absence of a crystal structure, one possible

169 explanation is that the positively charged side-chain $\varepsilon$-amino group of DYRK1A $\mathrm{A}^{\mathrm{R} 28 \mathrm{~K}}$ has been

170 repositioned, and now facilitates a favorable interaction with substrates possessing an acidic

171 residue at position $\mathrm{P}+1$.

173 Double mutation of residues Q323 and R328 completely reprograms DYRK1A P+1

\section{4 specificity}

175 Given the potentially independent influence of each of the DYRK1A $A^{\text {Q323G }}$ and DYRK1A $A^{\text {R328K }}$

176 mutations on the substrate $\mathrm{P}+1$ specificity, we decided to investigate if the double mutant

177 (referred to herein as DYRK1A ${ }^{Q R-G K}$ ) would more fully recapitulate CK II specificity at $P+1$ than

178 either individual mutant. In total, DYRK1A $\mathrm{A}^{\mathrm{QR}-\mathrm{GK}}$ ProPeL experiments led to the identification of

1791,535 unique phosphorylation sites (793 pSer, 645 pThr, and 97 pTyr). Together, the two 
180 mutations exhibited a combined effect on specificity, resulting in the complete reprogramming of

181 the $\mathrm{P}+1$ position from proline (characteristic of DYRK1A ${ }^{\mathrm{WT}}$ ) to acidic residue preferences

182 (mimicking CK II, Fig. 3C and Fig. S7C). Indeed, proline completely shifted from being the most

183 dominant feature of $D Y R K 1 A^{W T}$ at position $P+1$ to statistical underrepresentation in

184 DYRK1A $A^{Q R-G K}$. Interestingly, the effect of the double mutant on substrate preference appears

185 largely localized to the $\mathrm{P}+1$ position, and maintains the upstream basic as well as the $\mathrm{P}+2$

186 hydrophobic preferences of DYRK1A ${ }^{\text {WT }}$.

\section{In silico differential analysis offers additional insight}

189 Although differences between the kinase specificity motifs for DYRK1A variants can be

190 observed by simple inspection of the respective pLogos, it can be difficult to reconcile relative

191 deviations from the wild-type kinase, especially when the sizes of the foreground datasets vary.

192 To facilitate the direct comparison of each mutant DYRK1A with the wild-type kinase, we

193 performed an in silico differential analysis between the DYRK1A ${ }^{W T}$ and mutant DYRK1A data

194 sets. Typically pLogos will highlight significant motifs in kinase substrates by using

195 phosphorylation sites as a foreground data set, and amino acid frequencies from the organism's

196 proteome to determine background probabilities. In order to directly compare each DYRK1A

197 mutant to DYRK1 $A^{\text {WT }}$, we still used each mutant DYRK1A data set as the foreground, but then

198 used our 6,059 DYRK1A $1 \mathrm{WT}^{\mathrm{WT}}$ phosphorylation sites instead of the $E$. coli proteome as a

199 background data set. The resulting "differential pLogos" display residues that are over- and

200 underrepresented in the respective mutant DYRK1A substrate pool relative to the wild-type

201 kinase (DYRK1A $\left.{ }^{\mathrm{WT}}\right)$ substrates, rather than the background proteome.

202 As already noted, DYRK1A ${ }^{\text {Q323G }}$ differs from wild-type by favoring proline less than

203 DYRK1A $1 \mathrm{WT}^{\mathrm{WT}}$ at $\mathrm{P}+1$ and shifting $\mathrm{P}+1$ preference to alanine, and in the differential pLogo, that 
204 shift is abundantly clear (Fig. 3D and Fig. S7D). Next, the differential pLogo for DYRK1A $A^{\text {R328K }}$

205 (Fig. 3E and Fig. S7E) indicates no shift to disfavor proline at $P+1$ relative to DYRK1A ${ }^{W T}$, which

206 further confirms the standard pLogo results. A striking feature of the differential pLogo (that is

207 less obvious in the standard pLogo) is an overrepresentation of acidic residues with a

208 concomitant underrepresentation of basic residues at $\mathrm{P}+1$ compared to DYRK1A ${ }^{\mathrm{WT}}$. For

209 DYRK1 $A^{Q R-G K}$, the differential pLogo confirms the additive effects of the two mutants, namely

210 the disfavoring of proline and the favoring of acidic residues at the P+1 position. We also

211 observed that while arginine remains a positive determinant in the upstream positions for all

212 DYRK1A variants (Fig. 3A to 3C, and Fig. S7A to S7C), the importance of this upstream region

213 increases slightly but not uniformly in the mutants (note the more significant P-2 arginine in

214 differential pLogos Fig. 3E and Fig. S7D, S7E, but not in Fig. 3D, Fig. 3F nor Fig. S7F). This

215 suggests that in addition to reprogramming the $\mathrm{P}+1$ substrate preference, there may be some

216 subtle rearrangement of the kinase pocket, resulting in the observed shift in upstream basophilic

217 preferences.

\section{DISCUSSION}

220 Recently, catalytic domain kinase mutations associated with cancer have been linked to altered

221 substrate specificity, and modulated catalytic activity (5). While there have been previous

222 efforts at engineering kinases within the bacterial two-component system $(21,22)$, identification

223 of important residues influencing phosphoacceptor preference $(7,8)$, and tracing evolutionary

224 lineage through ancestral kinases (6), these studies have all exploited natural variation among

225 kinases. In the present study, we rationally engineered DYRK1A to first abolish its endogenous

$226 \mathrm{P}+1$ proline preference (which interestingly most closely resembles the specificity of the

227 unrelated kinase PKA). By incorporating a second point mutation to make our DYRK1A ${ }^{\text {QR-GK }}$ 
228 double mutant, we successfully shifted $\mathrm{P}+1$ preference to favor acidic residues and disfavor

229 proline. This combination results in a completely synthetic hybrid specificity that combines both

230 upstream DYRK1A and CK II P+1 preferences.

231 In large part, this investigation was made possible by the unprecedented resolution

232 achievable through the use of ProPeL, which provides an extremely detailed motif

233 representation that extends far beyond what is available from known endogenous substrates. It

234 is readily apparent that our approach can be scaled up to a more systematic interrogation of

235 structure-function relationships of other residues within the activation segment. The observation

236 that the DYRK1A ${ }^{\text {R328K }}$ mutant (CK II mimic) began to exhibit canonical CK II substrate

237 preferences, while the DYRK1 $A^{Q 323 G}$ mutant reduced proline preference by a seemingly distinct

238 mechanism, demonstrates that there is some independence of residues within the activation

239 segment, and it may be possible to program other effects without crosstalk. Such an

240 investigation would produce critical insights into the mechanistic underpinnings of kinase

241 specificity and the residues relevant to kinase-substrate interactions.

242 Exploring the mutation space of the activation segment may also help define the

243 sensitivity of protein kinases to mutational burden (23). As the number of disease-associated

244 missense mutations that localize to the catalytic domain continue to rise $(12,24,25)$, our

245 approach provides a powerful system for identifying mutation-induced kinase specificity

246 rewiring. Ultimately, such analyses may be invaluable for drug design and for cancer

247 therapeutics that often result in drug-resistant somatic kinase mutations with unintended

248 consequences. 


\section{MATERIALS AND METHODS}

\section{Plasmids, strains and in vivo proteome phosphorylation}

254 A plasmid containing the full-length coding sequences for the human DYRK1A gene in the 255 pDNR-Dual vector was purchased from the Harvard PlasmID Repository (Boston, MA). The 256 catalytic domain for DYRK1A (N137 - S496) was cloned into the pET45b vector (Novagen) by 257 traditional restriction site PCR cloning. All mutations were performed according to the

258 Stratagene QuickChange II protocol. DYRK1A constructs were expressed in the E. coli 259 OverExpress C41(DE3) strain (Lucigen) by IPTG induction. DYRK1A expression was optimal 260 when induced at mid-log with $0.5 \mathrm{mM}$ IPTG and incubated for 3 hours at $37^{\circ} \mathrm{C}$ with shaking at

$261250 \mathrm{rpm}$. Cells were harvested by centrifugation at $6,000 \mathrm{~g}, 4^{\circ} \mathrm{C}$ for 10 minutes, and stored 262 at $-80^{\circ} \mathrm{C}$.

264 Lysis and analysis of in vivo phosphorylation

265 Cell lysate was prepared as described previously $(9,26)$ with minor modifications. Cells were 266 lysed by sonication with a Fisher Sonic Dismembrator F60 at 15\% power using $15-20$ second 267 pulses, with 1 minute rest on ice between pulses, until lysate was clear. Crude lysate was 268 clarified by centrifugation at $20,000 \mathrm{~g}$ and $4^{\circ} \mathrm{C}$ for 30 minutes. Protein concentrations were 269 determined by Bichinchoninic Acid (BCA) Assay (Pierce), phosphorylation level was evaluated 270 by SDS-PAGE with Pro-Q Diamond Phosphoprotein stain (Life Technologies), and total protein 271 was evaluated by GelCode Blue coomassie staining (Life Technologies).

\section{Western Blotting}


274 Western blotting for DYRK1A used the primary antibody Anti-6xHis (NeuroMab clone N144/14,

275 RRID: AB_10671171, UC Davis/NIH NeuroMab Facility) at 1:1000 dilution, and IRDye 800CW

276 Goat anti-Mouse IgG secondary antibody (LI-COR Biosciences) at 1:5000 dilution.

278 In solution tryptic digestion

279 Samples were reduced, alkylated, digested with trypsin (Sequencing grade modified, Promega;

280 bovine trypsin, Sigma; or TrypZean, Sigma) at a 1:100 enzyme:substrate ratio, and desalted

281 with either $100 \mathrm{mg}$ or $500 \mathrm{mg} \mathrm{tC} 18$ SepPak Vac solid-phase extraction cartridges (Waters) as

282 previously described in Villén and Gygi, steps 2-17 (26). Desalted peptides were snap-frozen in

283 liquid nitrogen and lyophilized. Peptides were resuspended in appropriate buffer for one or

284 more of the following phosphopeptide enrichment strategies.

\section{PHOSPHOENRICHMENT}

287 Over the course of many mass spectrometry runs, many different phosphoenrichment strategies

288 were evaluated. Ultimately, we concluded that the most efficient sample preparation was a 289 simple $\mathrm{TiO}_{2}$ enrichment step, as described below. However, data from the other methods were 290 collected and accumulated for the DYRK1A ${ }^{\text {WT }}$ pLogo, and as such is summarized below. All 291 mutant DYRK1A data were obtained using simple bulk $\mathrm{TiO}_{2}$ enrichment.

\section{$293 \mathrm{TiO}_{2}$ bead enrichment.}

294 Phosphopeptide enrichment using bulk TiO2 beads (Titansphere $5 \mu \mathrm{m}$, GL Sciences) was

295 modified from Kettenbach and Gerber [16]. Beads were conditioned in bulk using Binding Buffer

296 (50\% ACN, 2 M Lactic Acid), with beads added at a 4:1 ratio to peptides (peptide concentration

297 estimated by NanoDrop A280 absorbance), and brought to a final peptide concentration of 
$2981 \mathrm{mg} / \mathrm{mL}$. Peptide/bead mix was incubated with maximum shaking on an Eppendorf

299 Thermomixer at room temperature for 1 hour. Beads were washed with Wash Buffer (50\% ACN,

$300 \quad 0.1 \%$ TFA) and eluted with $5 \% \mathrm{NH}_{4} \mathrm{OH}$. Eluate was immediately acidified by addition of $\mathrm{FA}$,

301 dried in a speed-vac, and stored at $-20^{\circ} \mathrm{C}$ for further enrichment, or analysis by mass

302 spectrometry.

\section{Strong Cation Exchange (SCX)}

305 Traditional SCX by HPLC was performed as described previously $(9,26)$. Separation by SCX-

306 SPE was performed according to Dephoure and Gygi (27). When SCX-SPE was performed

307 prior to $\mathrm{TiO}_{2}$ phosphoenrichment, fractions were desalted using $100 \mathrm{mg}$ tC18 SepPak Vac

308 cartridges. For SCX-SPE performed subsequent to $\mathrm{TiO}_{2}$ phosphoenrichment, fractions were

309 desalted using in-house StageTips (28) packed with 5 C18 discs per tip. Desalted samples

310 were dried in a speed-vac and stored at $-20^{\circ} \mathrm{C}$ for further enrichment, or analysis by mass

311 spectrometry.

313 Electrostatic Repulsion Hydrophilic Interaction Chromatography (ERLIC) by SPE.

314 ERLIC-SPE followed a similar principle as SCX-SPE, using the bulk material from ERLIC SPE

315 WAX Macrospin columns (The Nest Group). ERLIC-SPE columns were conditioned with

316 successive washes with methanol followed by water. Columns were incubated in

$3170.2 \mathrm{M} \mathrm{NaH}_{2} \mathrm{PO}_{4}, \quad 0.3 \mathrm{M} \mathrm{NaOAc}$ for $>1$ hour. Columns were equilibrated with $70 \% \mathrm{ACN}$,

$31820 \mathrm{mM} \mathrm{Na}-\mathrm{MePO}_{3}(\mathrm{pH} 2.0)$, and samples were loaded and washed with this same buffer.

319 Peptides were eluted sequentially first with $10 \% \mathrm{ACN}, 20 \mathrm{mM} \mathrm{Na}-\mathrm{MePO}_{3}(\mathrm{pH} 2.0)$, followed by

$32050 \mathrm{mM} \mathrm{NaH}_{2} \mathrm{PO}_{4}$. Final elution was achieved with $300 \mathrm{mM} \mathrm{NaH} \mathrm{PO}_{4}$. Eluates were desalted 
321 with either SepPak Vac cartridges or StageTips depending on volume, dried in a speed-vac and

322 stored at $-20^{\circ} \mathrm{C}$ for either further enrichment, or analysis by mass spectrometry.

323

\section{Peptide Identification by Tandem Mass Spectrometry}

325 Peptides were resuspended in $30 \mu \mathrm{L}$ Buffer A (3\% ACN, 0.125\% FA) and $14 \mu \mathrm{L}$ loaded onto a

326 C18 nanocapillary column with a pulled tip that sprays directly into the inlet of a Thermo Fisher

327 Scientific LTQ Orbitrap XL mass spectrometer. Peptides were eluted using an Agilent 1200

328 HPLC binary pump with a gradient that changes solvents from $100 \%$ to $65 \%$ Buffer A $(0 \%$ to

$32935 \%$ Buffer $B$ ) over a 48,85 , or 145 minute time period, where Buffer $A=3 \% A C N, 0.125 \%$ FA

330 in water, and Buffer B $=0.125 \%$ FA in ACN. A TOP10 method was used (MS scans followed by

331 Collision Induced Dissociation MS/MS on the top 10 most intense MS spectral peaks). Spectra

332 were searched using SEQUEST against the E. coli proteome, including decoy database entries,

333 which allowed for differential serine, threonine, and tyrosine phosphate modifications

334 (+79.966331), a differential methionine oxidation modification (15.9949146221) and a constant

335 cysteine modification of +57.02146374 . The deltaXCORR (the difference between the first and

336 second hits to the databases) was set to be $>=0.08$. To minimize false positives, for each of the

337 two classes of peptide charges $z=+2$ and $z>=+3$, XCORR thresholds were chosen to accept

338 peptides in such a manner that $1 \%$ of them were hits from the decoy database, resulting in an

339 expected False Discovery Rate (FDR) of $2 \%$.

\section{$341 \quad$ Phosphopeptide list filtering}

342 Prior to motif analysis, a master negative control list was generated by pooling phosphopeptides

343 previously identified in negative control experiments (9), previously identified endogenous E. coli

344 phosphorylation sites $(10,29)$, and phosphorylation sites identified in empty vector and kinase 
345 dead negative control experiments. Phosphorylation sites on this master negative control list

346 were removed from each active DYRK1A variant data set to generate a final list of kinase-

347 specific phosphorylation sites. Peptide lists from all runs were merged within each kinase

348 variant, and redundant peptides were removed prior to motif analysis.

350 pLogo Generation

351 To generate graphical motifs, known as pLogos, we used the online tool at plogo.uconn.edu,

352 previously described in detail (11). See Supporting Information for a more detailed explanation,

353 and instructions for recreating pLogos with our provided data.

354

\section{Scoring known kinase substrates}

356 scan- $x$ analyses of known and random substrates were carried out using an internal version of

357 the scan-x software (30). Candidate peptides were scored for a goodness-of-fit using our 358 DYRK1A ${ }^{\text {WT }}$ position weight matrix (PWM) obtained through ProPeL. Known verified human 359 substrates were retrieved from the PhosphoSitePlus database (12) (http://phosphosite.org), 360 while random substrates were obtained by randomly choosing an equivalent number of 361 serine/threonine 15 mers from the human proteome. Note that any substrate which was unable 362 to be extended to a P-site centered 15 mer due to proximity to either the $\mathrm{N}$ - or $\mathrm{C}$-terminus was 363 unable to be scored.

\section{5 motif-x Analysis}

366 motif-x analyses were carried out using an internal version of the motif-x web tool (19) with the

367 following parameters selected: central residue $=S^{*}, T^{*}$, or $Y^{*}$, width $=15$, foreground occurrence 
368 threshold $=5$, significance threshold $=0.00001$, background database $=$ NCBI E. coli proteome,

369 and background central residue $=\mathrm{S}, \mathrm{T}$, or $\mathrm{Y}$.

371 Structural Modeling

372 All structural modeling was visualized using PyMol for Mac, and using PDB Model 2WO6,

373 retrieved from the RCSB Protein Data Bank (14).

374

\section{SUPPLEMENTARY MATERIALS}

376 Fig. S1. pLogos for CK II, curated from known literature sites.

377 Fig. S2. Western blot for all DYRK1A kinase variants.

378 Fig. S3. SDS-PAGE gels for all DYRK1A kinase variants, using Pro-Q Diamond staining for

379 phosphorylation activity, and normalized to total protein Coomassie staining.

380 Fig. S4. Additional pLogos for DYRK1A ${ }^{\mathrm{WT}}$, including pLogos for threonine- and tyrosine-

381 centered substrates (curated from known literature sites, and ProPeL experiments, and pLogos

382 for a smaller subset of the data.

383 Fig. S5. Additional pLogos for DYRK1A ${ }^{\mathrm{WT}}$ with different positions "fixed" to show conditional

384 probabilities, and demonstrate multiple position correlations.

385 Fig. S6. Average position weight matrix (PWM) scores using the DYRK1A ${ }^{W T}$ pLogo to score

386 substrates of DYRK1A, non-CMGC family kinases, or random phosphoacceptors, curated from

387 the literature.

388 Fig, S7. Additional threonine-centered pLogos for mutant DYRK1A kinase variants.

389 Table S1. Mass spectrometry data.

390 Table S2. motif- $x$ runs

391 Table S3. Aligned data 


\section{REFERENCES AND NOTES}

393 1. T. Hunter, Signaling-2000 and Beyond. Cell. 100, 113-127 (2000).

394 2. T. Pawson, J. D. Scott, Protein phosphorylation in signaling -50 years and counting.

395 Trends Biochem. Sci. 30, 286-290 (2005).

3. L. A. Pinna, M. Ruzzene, How do protein kinases recognize their substrates? Biochim. Biophys. Acta BBA - Mol. Cell Res. 1314, 191-225 (1996).

4. J. C. Obenauer, L. C. Cantley, M. B. Yaffe, Scansite 2.0: proteome-wide prediction of cell signaling interactions using short sequence motifs. Nucleic Acids Res. 31, 3635-3641 (2003).

5. P. Creixell et al., Kinome-wide Decoding of Network-Attacking Mutations Rewiring Cancer Signaling. Cell. 163, 202-217 (2015).

6. C. J. Howard et al., Ancestral resurrection reveals evolutionary mechanisms of kinase plasticity. eLife. 3, e04126 (2014).

7. C. Chen et al., Identification of a Major Determinant for Serine-Threonine Kinase

8. P. Creixell et al., Unmasking Determinants of Specificity in the Human Kinome. Cell. 163, Phosphoacceptor Specificity. Mol. Cell. 53, 140-147 (2014). 187-201 (2015).

9. M. F. Chou et al., Using Bacteria to Determine Protein Kinase Specificity and Predict Target Substrates. PLoS ONE. 7, e52747 (2012).

10. B. Macek et al., Phosphoproteome Analysis of E. coli Reveals Evolutionary Conservation of

11. J. P. O'Shea et al., pLogo: a probabilistic approach to visualizing sequence motifs. Nat. Methods. 10, 1211-1212 (2013).

12. P. V. Hornbeck et al., PhosphoSitePlus, 2014: mutations, PTMs and recalibrations. Nucleic Acids Res. 43, D512-D520 (2015).

13. S. Himpel et al., Specificity Determinants of Substrate Recognition by the Protein Kinase DYRK1A. J. Biol. Chem. 275, 2431-2438 (2000).

14. M. Soundararajan et al., Structures of Down Syndrome Kinases, DYRKs, Reveal Mechanisms of Kinase Activation and Substrate Recognition. Structure. 21, 986-996 (2013).

15. N. Kannan, A. F. Neuwald, Evolutionary constraints associated with functional specificity of the CMGC protein kinases MAPK, CDK, GSK, SRPK, DYRK, and CK2 $\alpha$. Protein Sci. 13, 2059-2077 (2004). 
16. N. R. Brown, M. E. M. Noble, J. A. Endicott, L. N. Johnson, The structural basis for specificity of substrate and recruitment peptides for cyclin-dependent kinases. Nat. Cell Biol. 1, 438-443 (1999).

17. F. Meggio, O. Marin, L. A. Pinna, Substrate specificity of protein kinase CK2. Cell. Mol. Biol. Res. 40, 401-409 (1994).

18. P. A. Lochhead, G. Sibbet, N. Morrice, V. Cleghon, Activation-Loop Autophosphorylation Is Mediated by a Novel Transitional Intermediate Form of DYRKs. Cell. 121, 925-936 (2005).

19. D. Schwartz, S. P. Gygi, An iterative statistical approach to the identification of protein phosphorylation motifs from large-scale data sets. Nat. Biotechnol. 23, 1391-1398 (2005).

20. M. F. Chou, D. Schwartz, Curr. Protoc. Bioinforma. Ed. Board Andreas Baxevanis Al, in press, doi:10.1002/0471250953.bi1315s35.

21. J. M. Skerker et al., Rewiring the Specificity of Two-Component Signal Transduction Systems. Cell. 133, 1043-1054 (2008).

22. A. I. Podgornaia, P. Casino, A. Marina, M. T. Laub, Structural Basis of a Rationally Rewired Protein-Protein Interface Critical to Bacterial Signaling. Structure. 21, 1636-1647 (2013).

23. A. I. Podgornaia, M. T. Laub, Pervasive degeneracy and epistasis in a protein-protein interface. Science. 347, 673-677 (2015).

24. P. Lahiry, A. Torkamani, N. J. Schork, R. A. Hegele, Kinase mutations in human disease: interpreting genotype-phenotype relationships. Nat. Rev. Genet. 11, 60-74 (2010).

25. C. Ortutay, J. Väliaho, K. Stenberg, M. Vihinen, KinMutBase: A registry of disease-causing mutations in protein kinase domains. Hum. Mutat. 25, 435-442 (2005).

26. J. Villén, S. P. Gygi, The SCX/IMAC enrichment approach for global phosphorylation analysis by mass spectrometry. Nat. Protoc. 3, 1630-1638 (2008).

27. N. Dephoure, S. P. Gygi, A solid phase extraction-based platform for rapid phosphoproteomic analysis. Methods. 54, 379-386 (2011).

28. J. Rappsilber, Y. Ishihama, M. Mann, Stop and go extraction tips for matrix-assisted laser desorption/ionization, nanoelectrospray, and LC/MS sample pretreatment in proteomics. Anal. Chem. 75, 663-670 (2003).

29. N. C. Soares, P. Spät, K. Krug, B. Macek, Global Dynamics of the Escherichia coli Proteome and Phosphoproteome During Growth in Minimal Medium. J. Proteome Res. 12, 2611-2621 (2013).

30. D. Schwartz, M. F. Chou, G. M. Church, Predicting Protein Post-translational Modifications Using Meta-analysis of Proteome Scale Data Sets. Mol. Cell. Proteomics. 8, 365-379 (2009). 


\section{Acknowledgements}

461 The authors wish to thank Noah Dephoure and Craig Braun in the Gygi Lab for their assistance

462 and support with mass spectrometry experiments. We thank Sean Lubner, John Redden,

463 Stephanie Reeve, Megha Sah, Ben Stranges, and Randall Walikonis for generously sharing

464 reagents and/or providing invaluable technical advice. We also thank Prakhar Bansal for

465 maintainance and assistance with the Schwartz Lab computational tools. We thank Sean

466 Congdon, Lylah Deady, Fred Murphy, Benjamin Currall and Anastasios Tzingounis for helpful

467 discussions and the critical reading of our manuscript. Finally, we thank the University of

468 Connecticut Computational Biology Core for hosting the pLogo web site, and maintaining the

469 clusters on which it runs.

\section{Funding}

472 This work was supported in whole or in part by grants awarded to G.M.C from the Department

473 of Energy (DE-FG02-02ER63445), and to D.S. from the University of Connecticut Research

474 Foundation, the University of Connecticut Office of the Vice President for Research, and the

475 National Institute of Neurological Disorders and Stroke (1R21NS096516).

\section{Author Contributions}

478 J.M.L. and D.S. conceived of the study. J.M.L., M.F.C., and D.S. designed the experiments.

479 J.M.L. and M.F.C. performed the experiments and analyzed the data. G.M.C. and D.S. 480 contributed materials, resources, and analysis tools. J.M.L. wrote the manuscript. All authors 481 helped edit the final manuscript. 


\section{Competing Financial Interests}

484 The authors declare no competing financial interests. However, G.M.C.s complete list of

485 potential conflicts of interest are available at http://arep.med.harvard.edu/gmc/tech.html.

487 FIGURE LEGENDS

488 Fig. 1. Schematic overview of ProPeL. A kinase of interest is cloned, and expressed in E. coli.

489 Resulting bacterial phosphorylation is evaluated by SDS-PAGE with Pro-Q Diamond and

490 Coomassie staining. Lysate is digested, phosphoenriched and identified by tandem mass

491 spectrometry. Data sets are computationally analyzed with motif-x (19) and visualized with

492 pLogo (11).

494 Fig. 2. Crystal structure and high-resolution pLogos for DYRK1A ${ }^{\text {WT }}$. (A) Structural

495 visualization (PDB Model 2WO6 (14)) indicating putative hydrogen bond between the main-

496 chain oxygen of Q323 and a side chain nitrogen of R328 in the DYRK1A ${ }^{\mathrm{WT}}$ activation segment.

497 DYRK1A is colored in cyan, with peptide substrate in magenta. (B to $\mathbf{C})$ pLogos (11) illustrate

498 substrate preferences for DYRK1 $\mathrm{A}^{\mathrm{WT}}$, constructed from either (B) known literature-curated

499 substrates (12) or (C) from unbiased ProPeL experiments. Overrepresented residues are

500 displayed above the x-axis, underrepresented residues are below the $\mathrm{x}$-axis. The $n(f g)$ and

$501 n(\mathrm{bg})$ values at the bottom left of the pLogo indicate the number of aligned foreground and

502 background sequences used to generate the image, respectively. The red horizontal bars

503 correspond to $p=0.05$ (corrected for multiple hypothesis testing), and $y$-axis is logarithmic

504 scale. The grey box indicates a "fixed" residue. Additional pLogos for threonine-centered

505 substrates are in Fig. S4.

506 
507 Fig. 3. DYRK1A ${ }^{\text {QR-GK }}$ exhibits altered P+1 substrate specificity. (A to C) pLogos (as

508 described in Fig. 2) illustrate substrate preferences for (A) DYRK1A ${ }^{Q 323 G}$, (B) DYRK1A ${ }^{R 328 K}$, and

509 (C) DYRK1A 1 QR-GK. (D to F) Differential pLogos display the relative changes in substrate

510 specificity between DYRK1A mutants and DYRK1A ${ }^{W T}$ by using DYRK1A ${ }^{\text {WT }}$ as a background

511 data set, and mutant foreground data sets were used for (D) DYRK1A ${ }^{Q 323 G}$, (E) DYRK1A ${ }^{R 328 K}$,

512 and (F) DYRK1A ${ }^{\text {QR-GK }}$. Additional pLogos for threonine-centered substrates in Fig. S7. 
bioRxiv preprint doi: https://doi.org/10.1101/091892; this version posted December 5, 2016. The copyright holder for this preprint (which was not certified by peer review) is the author/funder, who has granted bioRxiv a license to display the preprint in perpetuity. It is made available under aCC-BY 4.0 International license.

Figure 1

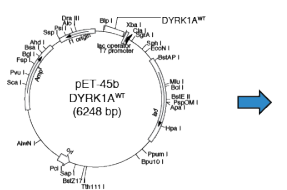

Clone Kinase of Interest

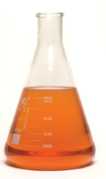

Express in E. coli

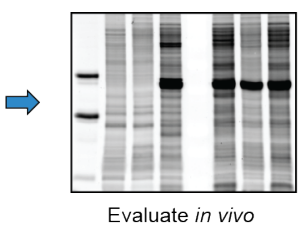

Phosphorylation

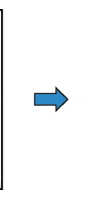

Tryptic Digestion

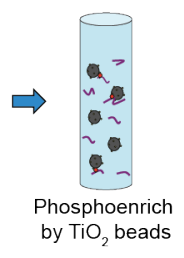

$\sqrt{3}$

AAADILRS * LYVDTAP AAEKDKVS * NQQDDMT

AAGLADS PLLEDID

AAMSGMLS * PELKQQI

AAPLAAFs * QIRHEVP

AAQEEEFs * LELRNRD

Create Foreground

Data Set

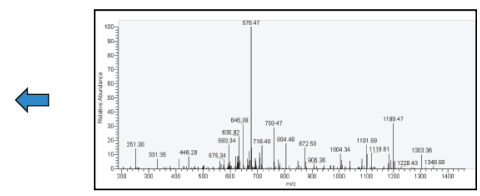

Identify Phosphopeptides by Tandem Mass Spectrometry 
bioRxiv preprint doi: https://doi.org/10.1101/091892; this version posted December 5, 2016. The copyright holder for this preprint (which was not certified by peer review) is the author/funder, who has granted bioRxiv a license to display the preprint in perpetuity. It is made available under aCC-BY 4.0 International license.

\section{Figure 2}

\section{A}

DYRK1A ${ }^{W T}$

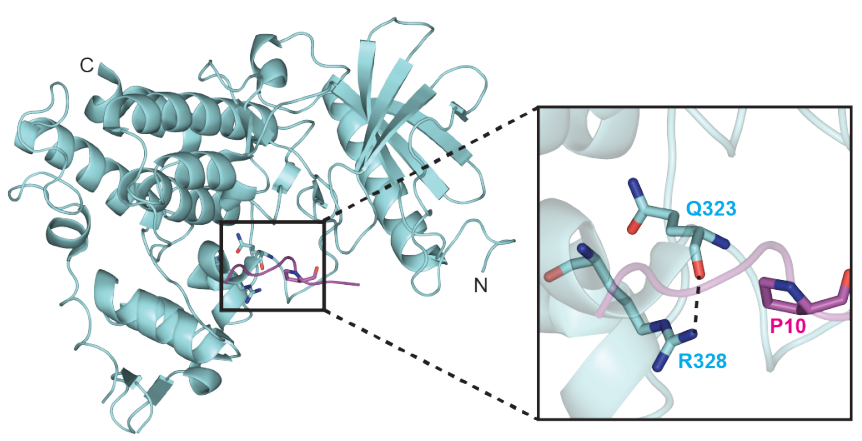

B DYRK1A ${ }^{\text {WT }}$ - Literature (Human Background)

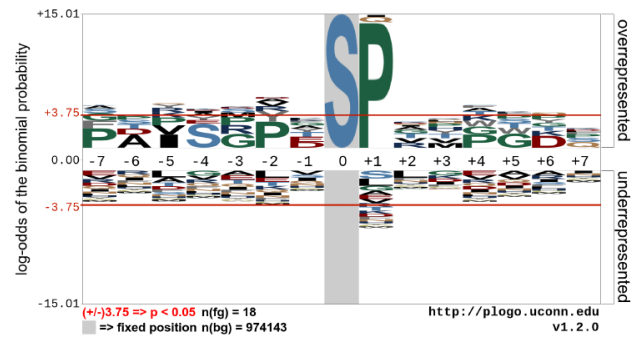

C DYRK1A ${ }^{\text {WT }}$ - ProPeL (E. coli Background)

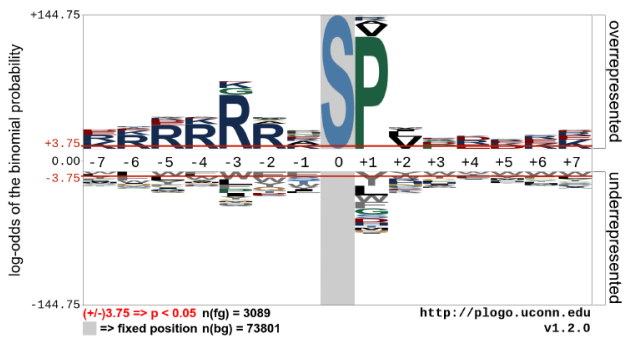




\section{Figure 3}

A

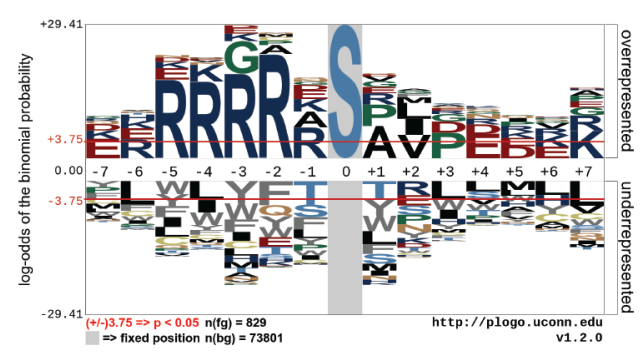

B

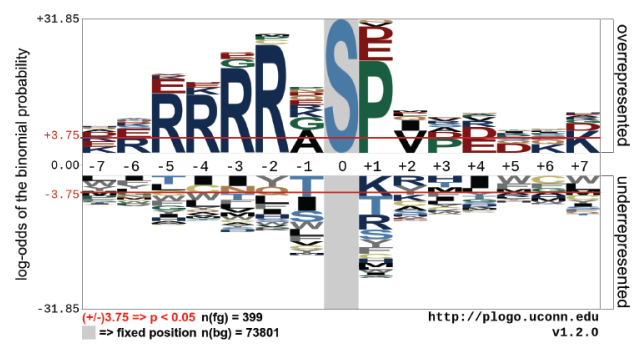

C

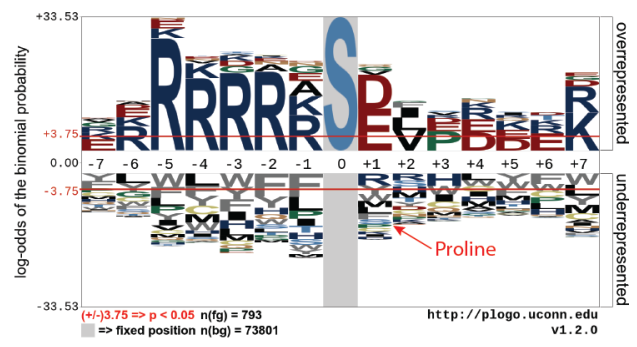

D DYRK1A ${ }^{Q 3236}$ (DYRK1A ${ }^{W T}$ Background)

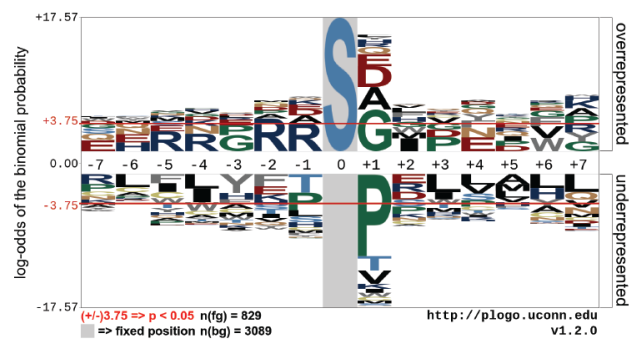

E DYRK1A $1 A^{R 28 K}$ (DYRK1A ${ }^{\text {WT }}$ Background)

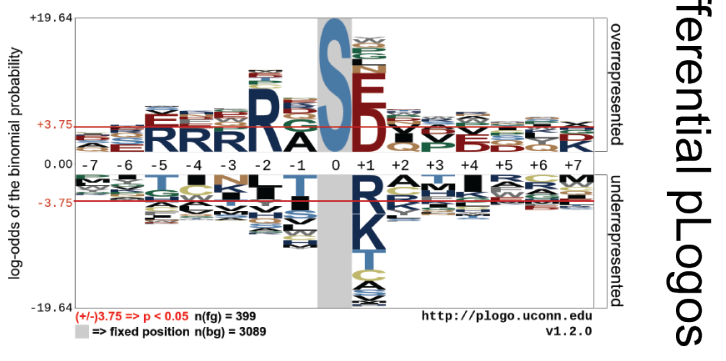

F DYRK1A $1 \mathrm{AR}^{\mathrm{QR}-\mathrm{GK}}\left(\mathrm{DYRK} 1 \mathrm{~A}^{\mathrm{WT}}\right.$ Background)

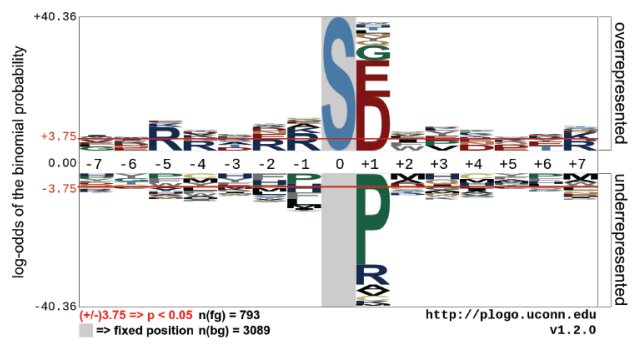

